\title{
EVALUACIÓN DE MARCADORES ANTROPOMÉTRICOS DE RIESGO CARDIOMETABÓLICO EN ADULTOS DE UNA COMUNIDAD DE LA REGIÓN CAÑADA DE OAXACA, MÉXICO.
}

\author{
EVALUATION OF ANTHROPOMETRIC INDEXES FOR CARDIOMETABOLIC RISK IN ADULTS OF A \\ COMMUNITY IN THE CAÑADA REGION OF OAXACA, MÉXICO.
}

\author{
Cruz-Sánchez Jacob J. *, Jiménez-Pineda Remedios*, Gutiérrez- Moguel Nelly V. *, Acosta- Chí Zaydi A.*, \\ Regalado- Santiago Citlalli*, González-Cano Patricia. \\ * Universidad de la Cañada, Oaxaca. México.
}

\section{RESUMEN}

Introducción: Las enfermedades cardiometabólicas representan un importante problema de salud pública a nivel mundial. La evaluación del riesgo permitiría una intervención médica y nutricional oportuna. Objetivo: Determinar la prevalencia de riesgo cardiometabólico (RCM) mediante marcadores antropométricos y la asociación entre dichos marcadores, en adultos de Teotitlán de Flores Magón, Oaxaca; México. Material y Método: Se realizó un estudio transversal, descriptivo en 208 individuos mayores de 20 años. El RCM se evaluó utilizando el índice de masa corporal, circunferencia de cintura, índice cintura-talla e índice de conicidad. Las variables se analizaron mediante estadística descriptiva; las pruebas de Chi-cuadrado de Pearson y de correlación de Spearman se utilizaron para las asociaciones y correlaciones respectivamente, considerando un nivel de significancia estadística de $\mathrm{P}<0.05$. Resultados: La prevalencia de obesidad fue mayor en mujeres $(42.34 \%)$ que en hombres (35.21\%). La prevalencia de RCM varió de $17.31 \%$ a $87.5 \%$, según el marcador utilizado, encontrando correlaciones positivas significativas entre todos ellos. Las mujeres presentaron mayor prevalencia de RCM en todos los marcadores evaluados. Conclusiones: Los hallazgos sugieren que la antropometría es una alternativa económica viable para detectar RCM en comunidades cuyos recursos en salud son limitados, sin embargo, podría complementarse con otro tipo de marcadores.

Palabras Clave: Riesgo cardiometabólico, circunferencia de cintura, índice cintura-talla.

\section{ABSTRACT}

Introduction: Cardiometabolic disease is an important public health problem worldwide, the early evaluation of risk factors will allow a prompt medical and nutritional intervention. Objective: To determine the prevalence of cardiometabolic risk (CMR) using anthropometry, and the associations between its indexes, in adults from Teotitlán de Flores Magón, Oaxaca; Mexico. Material and method: A descriptive, cross-sectional study was performed in 208 individuals older than 20 years old. CMR was evaluated based on body mass index, waist circumference, waist to height ratio and conicity index. All variables were analyzed using descriptive statistics; Pearson's Chi square and Spearman's correlation were used to analyze associations and correlations, respectively, $\mathrm{P}<0.05$ was considered significant. Results: The prevalence of obesity was significantly higher in women (42.34\%) than in men (35.21\%). Prevalence for CMR ranging from $17.31 \%$ to $87.5 \%$ was observed according to the parameter analyzed, a significant positive correlation was found among all parameters. A higher CMR among women for all markers analyzed was found. Conclusions: Our findings suggest that anthropometric measurements constitute an affordable alternative to evaluate the CMR on communities lacking health infrastructure, however, this approach could be completed using some other indicators.

Key words: Cardiometabolic risk, waist circumference, waist to height index

Correspondencia: Jacob J. Cruz Sánchez jacobjcs@unca.edu.mx

Recibido: 05 de febrero 2021, aceptado: 26 de mayo 2021

(C) Autor2021

(c) (1)

DOI: https://doi.org/10.29105/respyn20.3-2

Citation: Cruz-Sánchez J. J., Jiménez-Pineda R., Gutiérrez- Moguel N.V., Acosta- Chí Z.A., Regalado-

Santiago C., González-Cano P. (2021) Evaluación de marcadores antropométricos de riesgo cardiometabólico en adultos de una comunidad de la región Cañada de Oaxaca, México. Revista Salud Pública y Nutrición, 20 (3), 8-17. 


\section{Introducción}

La obesidad es una enfermedad crónica multifactorial actualmente considerada como un problema importante de salud pública a nivel mundial debido a su incremento en décadas recientes (Wilson, 2020). Es el resultado de prácticas y factores de riesgo que pueden ser de carácter inmediato (a nivel individual), intermedio (en el entorno de los individuos), y básicos o estructurales (a nivel macro) (Rivera-Dommarco et al., 2019). La obesidad es definida como el aumento desproporcionado del peso corporal con una acumulación excesiva de tejido adiposo (GonzálezMuniesa et al., 2017), causada principalmente por el balance energético positivo (ingesta calórica elevada y/o disminución del gasto energético) (San-Cristobal et al., 2020). La acumulación crónica de grasa corporal promueve el desarrollo de inflamación crónica de bajo grado debido al desbalance en el control de la homeostasis energética (Charakida et al., 2014). La obesidad se encuentra asociada al desarrollo de hipertensión arterial, dislipidemia, enfermedades cardiovasculares, ciertos tipos de cáncer, apnea del sueño, diabetes mellitus tipo 2 (DMT2), entre otras (González-Muniesa et al., 2017; Marcos-Delgado et al., 2020).

Actualmente existen diversos métodos para diagnosticar la obesidad, en los que se incluyen el análisis de impedancia bioeléctrica (BIA), absorciometría dual de rayos $X$ (DEXA), densitometría, tomografía computarizada, resonancia magnética, y la antropometría (Fosbøl \& Zerahn, 2015). Ésta última ha mostrado ser una opción viable debido a su rapidez, sencillez y accesibilidad (Ramírez-Vélez et al., 2019). En este sentido, el índice de masa corporal (IMC), determinado como el peso corporal $(\mathrm{kg})$ dividido entre la talla al cuadrado $(\mathrm{m} 2)$, es el método más utilizado en todo el mundo, a pesar de ser impreciso (Javed et al., 2015). La Organización Mundial de la Salud (OMS) clasifica al sobrepeso y la obesidad con base a los valores del IMC, asignando para cada uno de ellos valores de $25-29.9 \mathrm{~kg} / \mathrm{m} 2 \mathrm{y} \geq 30 \mathrm{~kg} / \mathrm{m} 2$, respectivamente (Després, 2012).

Debido a que el IMC por sí solo no indica el nivel de adiposidad central, de forma complementaria, se sugiere la evaluación de otros marcadores antropométricos que ayuden a establecer el riesgo cardiometabólico (RCM), como la circunferencia de cintura (CC) (Ying et al., 2010). El RCM hace referencia a los factores de riesgo que aumentan la probabilidad de experimentar eventos vasculares o desarrollar diabetes, dentro de estos, no únicamente se contemplan marcadores antropométricos (ej. CC), sino que incluye otros elementos como el tabaquismo, la etnia, los antecedentes heredofamiliares, el género, los niveles de lípidos en sangre, entre otros (Chatterjee et al., 2012). La CC es un método simple para evaluar la adiposidad abdominal que puede ser aplicado en la clínica (Ross et al., 2020). Alrededor del mundo se han propuesto otros marcadores antropométricos de RCM como el índice cintura - talla (ICT), así como el índice de conicidad (ICO), sin embargo, aún existe controversia con respecto a qué marcadores antropométricos tienen mayor utilidad en la predicción de RCM (Browning et al., 2010). Se ha sugerido que un ICT $\geq 0.5$ puede predecir RCM elevado, independientemente de la edad, sexo o etnia (Graves et al., 2014). Por otro lado, el ICO se ha considerado útil para la evaluación de la obesidad central y ha sido estudiado como un índice factible en la predicción de alteraciones en los niveles de insulina en ayunas, presión arterial y niveles de triglicéridos en sangre (Mantzoros et al., 1996).

México atraviesa por una transición epidemiológica importante que incluye enfermedades crónicas no transmisibles y cambios importantes en el estilo de vida que predisponen al desarrollo de comorbilidades. A nuestro conocimiento, en la región Cañada no se ha reportado la evaluación de estos marcadores antropométricos en adultos para la detección oportuna de RCM. Por lo que, el presente estudio tuvo como objetivo determinar la prevalencia de RCM, así como evaluar la asociación entre los marcadores de RCM en adultos de la población de Teotitlán de Flores Magón, Oaxaca; México.

\section{Material y Método}

Diseño de estudio y sujetos de estudio

Se trató de un estudio transversal descriptivo realizado durante el periodo del 11 de noviembre del 2019 al 20 de enero del 2020, que incluyó a 208 adultos ambulatorios de 20 años o más, de ambos sexos, elegidos aleatoriamente, residentes de la comunidad de Teotitlán de Flores Magón, perteneciente a la Región Cañada de Oaxaca en México, el cual cuenta con una población total de 9,876 personas, de los cuales el $61 \%$ corresponde a 
adultos de 20 años o más, de acuerdo al censo INEGI 2015 (INEGI, 2015). Todos los participantes dieron su consentimiento informado para participar en el estudio. Los criterios de exclusión fueron personas menores de 20 años; mujeres embarazadas; personas con inhabilidad para mantenerse de pie; institucionalizadas $\mathrm{u}$ hospitalizadas; así como aquellas que se rehusaron a participar en el estudio al no dar su consentimiento informado.

\section{Antropometría}

El peso y la talla fueron determinados a través de métodos estándar establecidos por la OMS. Brevemente, la talla se midió con un estadímetro portátil (BAME EP AL, México) con el paciente de pie, sin zapatos, hombros relajados, con la mirada hacia adelante y la espalda hacia la pared (plano de Frankfort). El peso se midió con el paciente con ropa ligera (cuando fue posible), utilizando un analizador de composición corporal (TANITA fit Scan, Japón).

El IMC fue determinado dividiendo el peso en $\mathrm{kg}$ entre la talla (m) al cuadrado. La CC fue medida utilizando una cinta métrica (Lufkin, USA) con el paciente en posición vertical, en el punto medio entre la parte superior de la cresta iliaca derecha y el margen inferior de la última costilla, la cinta métrica (flexible, pero no estirable) se colocó de manera ajustada, pero sin hacer presión (y paralela al piso), sobre el abdomen, la lectura se tomó una vez que el paciente terminó de expirar; se estableció como puntos de corte de obesidad abdominal y RCM elevado una circunferencia de cintura $\geq 80 \mathrm{~cm}$ en mujeres y $\geq 90 \mathrm{~cm}$ en hombres (Shamah-Levy et al., 2019; Wall-Medrano et al., 2016; World Health Organization, 2008). El ICT se calculó dividiendo la circunferencia de cintura $(\mathrm{cm})$ y la talla $(\mathrm{cm})$. Se ha propuesto como punto de corte un valor $\geq 0.5$ tanto en hombres como en mujeres para establecer RCM (Aguilar-Morales et al., 2018; Yoo, 2016). El ICO, como ya se mencionó, es un índice de adiposidad abdominal y de RCM propuesto por Valdéz et al. en el año 1991 (Valdez, 1991) el cual fue calculado de la siguiente forma:

$$
I C O=\frac{C C(\mathrm{~cm})}{0.109 \sqrt{\frac{\text { Peso }(\mathrm{kg})}{\text { Talla }(\mathrm{m})}}}
$$

Aunque los puntos de corte pueden variar dependiendo de la población, se ha propuesto un punto de corte de $\geq 1.25$ en hombres $\mathrm{y} \geq 1.18$ en mujeres para establecer obesidad abdominal y RCM (Pitanga \& Lessa, 2005).

\section{Análisis estadístico}

La normalidad de las variables cuantitativas se determinó a través de la prueba de KolmogorovSmirnoff (Mishra et al., 2019). Los datos se muestran como media \pm desviación estándar (DE) o mediana [rango intercuartil (RIQ)]; las variables cualitativas se muestran como frecuencias y porcentaje. La evaluación de las diferencias de variables cuantitativas entre hombres y mujeres se realizó a través de la prueba t de Student o prueba de suma de rangos de Wilcoxon. La evaluación de la asociación entre marcadores antropométricos de RCM y el sexo se realizó a través de la prueba Chi-cuadrado (X2) de Pearson. Para la determinación de los coeficientes de correlación entre los marcadores antropométricos de RCM se utilizó la prueba de correlación de Spearman. Los análisis estadísticos fueron realizados utilizando el software STATA versión 14 (Collage Station, TX, USA) y R Studio versión 1.3.1093 (Foundation for Statistical Computing, Viena, Austria), ambas para Macintosh. Se consideró un valor de $\mathrm{P}<0.05$ como significancia estadística.

\section{Resultados}

De los 208 sujetos evaluados, 137 corresponden a mujeres $(65.86 \%)$, la mediana de edad de los sujetos de estudio fue de 37.50 (21) años; no se observaron diferencias estadísticamente significativas en la edad entre hombres y mujeres, así como en el IMC, CC e ICO (Tabla 1). El IMC promedio de los sujetos fue de $28.76 \pm 4.81(\mathrm{~kg} / \mathrm{m} 2)$. El ICT fue mayor mujeres que en hombres $(0.61 \pm 0.09$ vs $0.58 \pm 0.08$ respectivamente; $\mathrm{P}=0.0110)$. 
Tabla 1. Características generales de los sujetos de estudio por sexo

\begin{tabular}{lccc}
\hline \multirow{2}{*}{ Variables } & Total & Hombres & Mujeres \\
\cline { 2 - 4 } & $(\mathrm{n}=208)$ & $(\mathrm{n}=71)$ & $(\mathrm{n}=137)$ \\
\hline Edad $(\mathrm{años})^{\mathrm{a}}$ & $37.50(21)$ & $34(24)$ & $38(19)$ \\
Peso $(\mathrm{kg})^{a * * *}$ & $71.77 \pm 14.18$ & $77.30 \pm 12.68$ & $68.90 \pm 14.10$ \\
Talla $(\mathrm{m})^{\mathrm{b} * * *}$ & $1.56(0.13)$ & $1.65(0.08)$ & $1.54(0.07)$ \\
$\mathrm{IMC}\left(\mathrm{kg} / \mathrm{m}^{2}\right)^{\mathrm{b}}$ & $28.76 \pm 4.81$ & $28.07 \pm 4.00$ & $29.11 \pm 5.16$ \\
$\mathrm{CC}(\mathrm{cm})^{\mathrm{b}}$ & $94.15 \pm 13.16$ & $95.42 \pm 12.31$ & $93.49 \pm 13.58$ \\
$\mathrm{ICT}$ & $0.60 \pm 0.87$ & $0.58 \pm 0.08$ & $0.61 \pm 0.09$ \\
$\mathrm{ICO}^{\mathrm{a}}$ & $1.09(0.14)$ & $1.10(0.14)$ & $1.09(0.14)$ \\
\hline
\end{tabular}

Fuente: elaboración propia.

Datos mostrados como media \pm DE o mediana (RIQ). IMC: índice de masa corporal; CC: circunferencia de cintura; ICT: índice cintura-talla; ICO: índice de conicidad.

$* P<0.05$

*** $P<0.0001$

${ }^{a}$ Suma de rangos de Wilcoxon

${ }^{\mathrm{b}}$ t de Student

El 39.90\% de los sujetos presentó obesidad, siendo mayor la prevalencia en mujeres $(42.34 \%)$ que en hombres (35.21\%). De acuerdo con la CC, las mujeres presentaron mayor obesidad abdominal y RCM comparado con los hombres $(86.86 \%$ vs $74.65 \%$, respectivamente).

En cuanto al ICT, el $89.78 \%$ de las mujeres presentaron RCM, mientras que en hombres fue de $83.10 \%$. En lo que respecta al ICO, el $21.17 \%$ de las mujeres y el $9.86 \%$ de los hombres presentaron RCM. En el análisis de asociación entre variables cualitativas, encontramos asociaciones estadísticamente significativas entre el sexo y la CC $(\mathrm{P}<0.001)$, ICO $(\mathrm{P}=0.41)$, pero no para IMC e ICT (Tabla 2).

\begin{tabular}{|c|c|c|c|}
\hline & $\begin{array}{l}\text { Total } \\
\mathrm{n}(\%)\end{array}$ & $\begin{array}{c}\text { Hombres } \\
\text { n (\%) }\end{array}$ & $\begin{array}{c}\text { Mujeres } \\
\mathrm{n}(\%)\end{array}$ \\
\hline \multicolumn{4}{|l|}{ IMC } \\
\hline Normopeso & $50(24.04)$ & $17(23.94)$ & $33(24.09)$ \\
\hline Sobrepeso & $75(36.06)$ & $29(40.85)$ & $46(33.58)$ \\
\hline $\begin{array}{l}\text { Obesidad } \\
\text { ICT }\end{array}$ & $83(39.90)$ & $25(35.21)$ & $58(42.34)$ \\
\hline Sin riesgo & $26(12.50)$ & $12(16.90)$ & $14(10.22)$ \\
\hline Con riesgo & $182(87.50)$ & $59(83.10)$ & $123(89.78)$ \\
\hline \multicolumn{4}{|l|}{$\mathrm{CC}^{*}$} \\
\hline Sin riesgo & $37(17.79)$ & $19(26.76)$ & $18(13.14)$ \\
\hline $\begin{array}{l}\text { Con riesgo } \\
\text { ICO*}^{*}\end{array}$ & $171(82.21)$ & $52(73.24)$ & $119(86.86)$ \\
\hline Sin riesgo & $172(82.69)$ & $64(90.14)$ & $108(78.83)$ \\
\hline Con riesgo & $36(17.31)$ & $7(9.86)$ & $29(21.17)$ \\
\hline \multicolumn{4}{|c|}{ Fuente: elaboración propia } \\
\hline \multicolumn{4}{|c|}{$\begin{array}{l}\text { IMC: índice de masa corporal; CC: circunferencia de cintura; } \\
\text { ICT: índice cintura-talla; ICO: índice de conicidad. }\end{array}$} \\
\hline \multicolumn{4}{|c|}{ Chi-cuadrada de Pearson * $P<0.05$} \\
\hline
\end{tabular}

Los coeficientes de correlación de Spearman entre los marcadores de RCM se muestran en la Tabla 3. Se encontraron correlaciones positivas significativas entre todos los marcadores evaluados. La correlación más fuerte se observó entre la CC y el ICO (rho = 0.9695; $\mathrm{P}<0.0001)$. Se observaron correlaciones positivas débiles estadísticamente significativas entre la edad y todos los marcadores antropométricos de RCM evaluados.

\begin{tabular}{|c|c|c|c|c|c|}
\hline & $\mathrm{CC}(\mathrm{cm})$ & $\begin{array}{c}\text { IMC } \\
\left(\mathrm{kg} / \mathrm{m}^{2}\right)\end{array}$ & ICT & ICO & $\begin{array}{c}\text { Edad } \\
\text { (años) } \\
\end{array}$ \\
\hline $\mathrm{CC}(\mathrm{cm})$ & 1 & - & - & - & - \\
\hline IMC $\left(\mathrm{kg} / \mathrm{m}^{2}\right)$ & $0.7744^{\star \star *}$ & 1 & - & - & - \\
\hline ICT & $0.9003^{* * *}$ & $0.7776^{\star \star \star *}$ & 1 & - & - \\
\hline ICO & $0.9695^{\star * *}$ & $0.6423^{* * *}$ & $0.9022^{\star \star *}$ & 1 & - \\
\hline Edad (años) & $0.3141^{\star \star *}$ & $0.2753^{\star \star \star}$ & $0.4228^{\star \star *}$ & $0.3642^{\star \star *}$ & 1 \\
\hline
\end{tabular}

\section{Discusión}

Las enfermedades cardiometabólicas representan actualmente un importante desafío para la salud pública a nivel mundial, por lo que es necesaria la detección temprana de RCM en adultos para proporcionar tratamientos de forma adecuada $\mathrm{y}$ oportuna. Diversos marcadores han sido propuestos para la determinación de RCM en adultos, que incluyen niveles disminuidos de colesterol HDL (Melin et al., 2019), hipertrigliceridemia (Sniderman 
et al., 2018), resistencia a la insulina (Ormazabal et al., 2018), presión arterial elevada (Fuchs \& Whelton, 2020) y obesidad abdominal (Smith \& Haslam, 2007). Sin embargo, la medición de biomarcadores en sangre, en ocasiones no es factible en el ámbito clínico, debido a la falta de insumos o el costo elevado que pueden representar. Por lo que, marcadores antropométricos han sido propuestos para evaluar la obesidad abdominal y el RCM (Dhana et al., 2015).

En México, la Encuesta Nacional de Salud y Nutrición (ENSANUT) 2018 100k (que contempla a localidades con menos de 100,000 habitantes), muestra una prevalencia de obesidad de $33.6 \%$ determinada mediante el IMC en adultos de ambos sexos de 20 años o más, siendo más prevalente en mujeres $(40.1 \%)$ que en hombres $(26.6 \%)$. En este trabajo se encontraron resultados similares, una prevalencia de $39.90 \%$ de obesidad en ambos sexos, siendo mayor en mujeres que en hombres $(42.34 \%$ vs 35.21, respectivamente). La comparación se realizó con la ENSANUT 2018 100k debido a que Teotitlán de Flores Magón, Oaxaca, corresponde a un municipio con menos de 100,000 habitantes (Shamah-Levy et al., 2019). Las mujeres presentan aproximadamente $10 \%$ más de grasa corporal comparado con los hombres. La edad, es un factor importante que incrementa la cantidad de grasa corporal en ambos sexos, sin embargo, las mujeres tienden a acumular mayor cantidad de grasa que los hombres en todas las etapas de la vida (Gallagher et al., 1996), especialmente después de la pubertad por efectos hormonales (Fitzgerald et al., 2018).

Es importante mencionar que, depende mucho la ubicación de la grasa corporal, mientras que las mujeres que acumulan grasa en regiones del cuerpo como cadera y muslos tienen menor riesgo de desarrollar enfermedades cardiometabólicas, en aquellas que acumulan grasa en la región abdominal, el riesgo de desarrollar dichas enfermedades incrementa de forma importante (Karastergiou et al., 2012).

En cuanto a la obesidad abdominal y RCM determinado mediante la $\mathrm{CC}$, se encontró una prevalencia del $82.21 \%$ en ambos sexos, siendo mayor en mujeres que en hombres $(86.86 \%$ vs $73.24 \%$, respectivamente). Estos datos se asemejan a lo reportado por la ENSANUT 2018 100k en donde a nivel nacional, se mostró una prevalencia de obesidad abdominal y RCM (determinado mediante CC) en ambos sexos del $75.6 \%$, siendo de igual forma las mujeres las más afectadas que los hombres (85.6\% vs $65.3 \%$ respectivamente (Shamah-Levy et al., 2019). En el 2005, un estudio realizado en una comunidad rural de la región Mixteca de Oaxaca (estudio no publicado), Fernández et al. observaron que el $39.5 \%$ de las mujeres en edad de 20 a 49 años de edad presentaron una $\mathrm{CC}>80 \mathrm{~cm}$, mientras que los hombres en el mismo rango de edad, $12.1 \%$ presentaron una CC > $90 \mathrm{~cm}$ (Arroyo et al., 2007).

Encontramos que el $87.5 \%(n=187)$ de los sujetos tuvo un ICT elevado $(\geq 0.5)$, siendo más prevalente en mujeres $(89.78 \%)$ que en hombres $(83.10 \%)$, estos datos muestran la gran prevalencia de RCM en sujetos adultos, que reafirman lo hallado en una muestra de adultos de la ENSANUT 2016 de medio camino, en donde se observó que el $91.4 \%$ de los participantes tuvieron un ICT elevado, siendo mayor en mujeres que en hombres (93.2\% vs $87.9 \%$, respectivamente) (Rangel-Baltazar et al., 2019). En cuanto al ICO, se observó que el $17.31 \%$ de los participantes presentaron RCM, siendo las mujeres más afectadas que los hombres $(21.87 \%$ vs 9.86 , respectivamente). Lo anterior, difiere de los otros marcadores evaluados, esto se puede explicar debido a la falta de consenso en los puntos de corte propuestos para la determinación de RCM. Esto abre un área de oportunidad a tomar en cuenta en futuras investigaciones.

Con respecto a las correlaciones, observamos que la CC se correlacionó positivamente con el IMC, concordando con otros trabajos, en donde se ha correlacionado la CC con el IMC positivamente en una población polaca con síndrome metabólico $(\mathrm{R}=$ $0.78, \mathrm{P}<0.01$ ) (Gierach et al., 2014), esto ha sido observado también en mujeres iraníes postmenopáusicas $(\mathrm{r}=0.67, \mathrm{P}<0.05)$ (Shidfar et al., 2012) y con sobrepeso u obesidad $(r=0.430, P<$ 0.001) (Ashtary-Larky et al., 2018). Es importante mencionar, que algunos trabajos han demostrado que el IMC puede tener una eficacia similar a la CC para predecir el desarrollo de enfermedades como diabetes mellitus tipo 2, entre otras alteraciones metabólicas (Gierach et al., 2014). Del mismo modo, otros estudios han reportados correlaciones positivas entre los marcadores antropométricos de RCM evaluados en este estudio, como lo reportado por 
Shidfar et al., quienes observaron en un estudio transversal en mujeres postmenopáusicas en Irán, correlaciones positivas entre el ICO e IMC $(r=0.31$, $\mathrm{P}<0.05)$ e ICO con CC $(\mathrm{r}=0.86, \mathrm{P}<0.05)$ (Shidfar et al., 2012). En un estudio transversal conducido por Zhang et al. en donde evaluaron a 67,334 mujeres sin historial enfermedades cardiovasculares, se halló una fuerte correlación positiva entre el ICO y el ICT ( $\mathrm{r}=$ 0.804), así como el ICO con el IMC ( $\mathrm{r}=0.800)$ (Zhang et al., 2004). Así mismo, Ashtary-Larky et al. reportó correlaciones positivas entre el IMC con el ICT $(r=0.465, \mathrm{P}<0.001)$, y CC con ICT $(r=0.986$, $\mathrm{P}<0.001)$ en mujeres iraníes con sobrepeso $\mathrm{u}$ obesidad (Ashtary-Larky et al., 2018).

Por otro lado, se encontró una correlación positiva estadísticamente significativa entre la edad y los marcadores de RCM, sin embargo, estas asociaciones, aunque significativas, fueron débiles. Diversos estudios han mostrado el aumento en los depósitos de grasa, especialmente en la región abdominal, a medida que aumenta la edad (Stevens et al., 2010). A manera de ejemplo, en un análisis transversal de la Encuesta de Salud Nacional y Examen de Nutrición (NHANES, por sus siglas en inglés) de los Estados Unidos, que incluyó mujeres y hombres, mostró que, en hombres la CC aumenta aproximadamente $13.4 \mathrm{~cm}$ en individuos de entre 2029 años, en comparación con individuos de entre 6069 años; en mujeres, las estimaciones fueron de 5.9 a $8.1 \mathrm{~cm}$ menos que los hombres, sin embargo, el aumento de la CC con respecto a la edad fue significativo (Ford et al., 2003; Stevens et al., 2010). El IMC suele aumentar considerablemente durante la edad adulta, este aumento se ha asociado con el aumento en la cantidad de grasa corporal como masa muscular (Welch \& Sowers, 2000), por lo que, como se mencionó, es una de las principales desventajas de la utilización del IMC por sí solo, para la determinación de RCM.

En este trabajo, encontramos asociaciones significativas solo entre el sexo y CC, e ICO. Estos resultados se encuentran en línea con lo reportado por Magchún-Hernández et al. en adolescentes mexicanos del estado de Chiapas, México, en donde observaron asociación significativa entre el sexo con CC ( $\mathrm{P}=0.005)$ (Megchún-Hernández et al., 2019), por otro lado, se ha encontrado correlación positiva entre el sexo femenino e ICO $(r=0.092, \mathrm{P}<0.001)$ y sexo masculino $(r=0.130, P<0.001)$ en un estudio transversal que incluyó sujetos adultos con riesgo cardiovascular de España (Sánchez et al., 2020).

A nuestro conocimiento, este es el primer trabajo que evalúa obesidad, obesidad abdominal, así como marcadores antropométricos de RCM en adultos en una comunidad de la región Cañada, en Oaxaca, México. Si bien, este trabajo, al aportar datos valiosos sobre el estado nutricional de la población de Teotitlán de Flores Magón, Oaxaca, también tiene ciertas limitaciones. Al ser un estudio transversal, únicamente se puede observar el comportamiento de las variables estudiadas, es decir, es imposible determinar la causalidad y temporalidad. Lo anterior, produjo una serie de cuestionamientos para poder entender mejor el curso de las enfermedades crónicas no transmisibles, y dar pautas para poder realizar estudios con mayor rigor metodológico.

\section{Conclusiones}

Dentro de la práctica clínica del profesional de salud, en ocasiones por causas económicas o falta de insumos no se puede realizar una evaluación bioquímica, por lo que la determinación de mediciones e indicadores antropométricos como la CC, el ICT y el ICO pueden ser una alternativa para establecer el RCM en la población.

En la comunidad evaluada, la prevalencia de RCM en adultos varía de acuerdo al marcador antropométrico utilizado, siendo mayor con la CC e ICT. Es importante hacer hincapié en que las mujeres presentaron una mayor prevalencia de RCM en todos los marcadores evaluados. Sin embargo, aunque las mediciones e indicadores antropométricos pueden ser una alternativa para establecer el RCM en la población, se sugiere el uso de otros marcadores para determinar la sensibilidad o especificidad de éstos.

Los resultados de la presente investigación ponen en relieve la importancia de desarrollar e implementar programas de prevención o disminución del RCM en la región de la Cañada Oaxaqueña, que consideren aspectos de educación nutricional, modificación del entorno alimentario, mejora en el acceso físico y económico de las familias para alcanzar una dieta saludable y sostenible así como transformar los factores que propicien la actividad física, con la finalidad de reducir la obesidad y sus comorbilidades. 


\section{Agradecimientos}

Agradecemos profundamente al $\mathrm{H}$. Ayuntamiento de Teotitlán de Flores Magón, Oaxaca, por los permisos proporcionados, y a la población, por su disponibilidad para participar en este estudio.

\section{Bibliografía}

Aguilar-Morales, I., Colin-Ramirez, E., RiveraMancía, S., Vallejo, M., \& Vázquez-Antona, C. (2018). Performance of waist-to-height ratio, waist circumference, and body mass index in discriminating cardio-metabolic risk factors in a sample of school-aged Mexican children. Nutrients, $\quad 10(12), \quad 1850$. https://doi.org/10.3390/nu10121850

Arroyo, P., Fernández, V., Loría, A., Pardío, J., Laviada, H., Vargas-Ancona, L., \& Ward, R. (2007). Obesidad, morfología corporal y presión arterial en grupos urbanos y rurales de Yucatán. Salud Publica de Mexico, 49(4), 274-285. https://doi.org/10.1590/S0036-

36342007000400007

Ashtary-Larky, D., Daneghian, S., Alipour, M., Rafiei, H., Ghanavati, M., Mohammadpour, R., Kooti, W., Ashtary-Larky, P., \& Afrisham, R. (2018). Waist circumference to height ratio: Better correlation with fat mass than other anthropometric indices during dietary weight loss in different rates. International Journal of Endocrinology and Metabolism, 16(4), 1. https://doi.org/10.5812/ijem.55023

Browning, L. M., Hsieh, S. D., \& Ashwell, M. (2010). A systematic review of waist-to-height ratio as a screening tool for the prediction of cardiovascular disease and diabetes: 05 could be a suitable global boundary value. Nutrition Research Reviews, 23(2), 247-269. https://doi.org/10.1017/S0954422410000144

Charakida, M., Khan, T., Johnson, W., Finer, N., Woodside, J., Whincup, P. H., Sattar, N., Kuh, D., Hardy, R., \& Deanfield, J. (2014). Lifelong patterns of BMI and cardiovascular phenotype in individuals aged 60-64 years in the 1946 British birth cohort study: An epidemiological study. The Lancet Diabetes and Endocrinology, 2(8), 648652. 8587(14)70103-2
Chatterjee, A., Harris, S. B., Leiter, L. A., Fitchett, D. H., Teoh, H., Bhattacharyya, O. K., \& Cardiometabolic Risk Working Group (Canadian). (2012). Managing cardiometabolic risk in primary care: summary of the 2011 consensus statement. Canadian Family Physician Medecin de Famille Canadien, 58(2), 389-393.

Després, J. P. (2012). Body fat distribution and risk of cardiovascular disease: An update. Circulation, 126(10), 1301-1313. https://doi.org/10.1161/CIRCULATIONAHA.1 11.067264

Dhana, K., Ikram, M. A., Hofman, A., Franco, O. H., \& Kavousi, M. (2015). Anthropometric measures in cardiovascular disease prediction: Comparison of laboratory-based versus non-laboratory-based model. Heart, 101(5), 377-383. https://doi.org/10.1136/heartjnl-2014-306704

Fitzgerald, S. J., Janorkar, A. V., Barnes, A., \& Maranon, R. O. (2018). A new approach to study the sex differences in adipose tissue. Journal of Biomedical Science, 89. https://doi.org/10.1186/s12929-018-0488-3

Ford, E. S., Mokdad, A. H., \& Giles, W. H. (2003). Trends in waist circumference among U.S. adults. Obesity Research, 11(10), 1223-1231. https://doi.org/10.1038/oby.2003.168

Fosbøl, M. O., \& Zerahn, B. (2015). Contemporary methods of body composition measurement. Clinical Physiology and Functional Imaging, 35(2), 81-97. https://doi.org/10.1111/cpf.12152

Fuchs, F. D., \& Whelton, P. K. (2020). High Blood Pressure and Cardiovascular Disease. In Hypertension (Vol. 75, Issue 2, pp. 285-292). Lippincott Williams and Wilkins. https://doi.org/10.1161/HYPERTENSIONAHA. 119.14240

Gallagher, D., Visser, M., Sepúlveda, D., Pierson, R. N., Harris, T., \& Heymsfieid, S. B. (1996). How useful is body mass index for comparison of body fatness across age, sex, and ethnic groups? American Journal of Epidemiology. https://doi.org/10.1093/oxfordjournals.aje.a0087 33 
Gierach, M., Gierach, J., Ewertowska, M., Arndt, A., \& Junik, R. (2014). Correlation between Body Mass Index and Waist Circumference in Patients with Metabolic Syndrome. ISRN Endocrinology, 2014(1), $1-6$. https://doi.org/10.1155/2014/514589

González-Muniesa, P., Mártinez-González, M. A., Hu, F. B., Després, J. P., Matsuzawa, Y., Loos, R. J. F., Moreno, L. A., Bray, G. A., \& Martinez, J. A. (2017). Obesity. Nature Reviews Disease Primers, https://doi.org/10.1038/nrdp.2017.34

Graves, L., Garnett, S. P., Cowell, C. T., Baur, L. A., Ness, A., Sattar, N., \& Lawlor, D. A. (2014). Waist-to-height ratio and cardiometabolic risk factors in adolescence: Findings from a prospective birth cohort. Pediatric Obesity, 9(5), 327-338. https://doi.org/10.1111/j.20476310.2013.00192.x

INEGI. (2015). Oaxaca. División municipal. INEGI. http://www.cuentame.inegi.org.mx/monografias/ informacion/oax/territorio/div_municipal.aspx?t ema $=$ me $\& e=20$

Javed, A., Jumean, M., Murad, M. H., Okorodudu, D., Kumar, S., Somers, V. K., Sochor, O., \& Lopez-Jimenez, F. (2015). Diagnostic performance of body mass index to identify obesity as defined by body adiposity in children and adolescents: A systematic review and metaanalysis. Pediatric Obesity, 10(3), 234-244. https://doi.org/10.1111/ijpo.242

Karastergiou, K., Smith, S. R., Greenberg, A. S., \& Fried, S. K. (2012). Sex differences in human adipose tissues - The biology of pear shape. Biology of Sex Differences, 3(1), 13. https://doi.org/10.1186/2042-6410-3-13

Mantzoros, C. S., Evagelopoulou, K., Georgiadis, E. I., \& Katsilambros, N. (1996). Conicity index as a predictor of blood pressure levels, insulin and triglyceride concentrations of healthy premenopausal women. Hormone and Metabolic Research, 28(1), 32-34. https://doi.org/10.1055/s-2007-979126
Marcos-Delgado, A., Fernández-Villa, T., MartínezGonzález, M. Á., Salas-Salvadó, J., Corella, D., Castañer, O., Martínez, J. A., Alonso-Gómez, Á. M., Wärnberg, J., Vioque, J., Romaguera, D., López-Miranda, J., Estruch, R., Tinahones, F. J., Lapetra, J., Serra-Majem, J. L., García-Molina, L., Tur, J. A., de Paz, J. A., ... Martín, V. (2020). The effect of physical activity and high body mass index on health-related quality of life in individuals with metabolic syndrome. International Journal of Environmental Research and Public Health, 17(10), 3728. https://doi.org/10.3390/ijerph17103728

Megchún-Hernández, M., Espinosa-Raya, J., GarcíaParra, E., Albavera-Hernández, C., \& BrionesAranda, A. (2019). Association of anthropometric indicators to evaluate nutritional status and cardiometabolic risk in mexican teenagers. Nutricion Hospitalaria, 36(5), 10491054. https://doi.org/10.20960/nh.02487

Melin, E. O., Thulesius, H. O., Hillman, M., Svensson, R., Landin-Olsson, M., \& Thunander, M. (2019). Lower HDL-cholesterol, a known marker of cardiovascular risk, was associated with depression in type 1 diabetes: A cross sectional study. Lipids in Health and Disease, 18(65). https://doi.org/10.1186/s12944-019$1009-4$

Mishra, P., Pandey, C. M., Singh, U., Gupta, A., Sahu, C., \& Keshri, A. (2019). Descriptive statistics and normality tests for statistical data. Annals of Cardiac Anaesthesia, 22(1), 67-72. https://doi.org/10.4103/aca.ACA_157_18

Ormazabal, V., Nair, S., Elfeky, O., Aguayo, C., Salomon, C., \& Zuñiga, F. A. (2018). Association between insulin resistance and the development of cardiovascular disease. Cardiovascular Diabetology, 17(122). https://doi.org/10.1186/s12933-018-0762-4

Pitanga, F. J. G., \& Lessa, I. (2005). [Anthropometric indexes of obesity as an instrument of screening for high coronary risk in adults in the city of Salvador--Bahia]. Arquivos Brasileiros de Cardiologia, 85(1), 26-31. https://doi.org//S0066-782X2005001300006 
Ramírez-Vélez, R., Pérez-Sousa, M. Á., Izquierdo, M., Cano-Gutierrez, C. A., González-Jiménez, E., Schmidt-Riovalle, J., González-Ruíz, K., \& Correa-Rodríguez, M. (2019). Validation of surrogate anthropometric indices in older adults: What is the best indicator of high cardiometabolic risk factor clustering? Nutrients, 11(8), 1701. https://doi.org/10.3390/nu11081701

Rangel-Baltazar, E., Cuevas-Nasu, L., ShamahLevy, T., Rodríguez-Ramírez, S., MéndezGómez-Humarn, I., \& Rivera, J. A. (2019). Association between high waist-to-height ratio and cardiovascular risk among adults sampled by the 2016 half-way national health and nutrition survey in Mexico (ENSANUT MC 2016). Nutrients, $11(6), \quad 1402$. https://doi.org/10.3390/nu11061402

Rivera-Dommarco, J. Á., Arantxa-Colchero, A. M., Luis-Fuentes, M., González de Cosío Martínez, T., Aguilar-Salinas, C. A., Hernández-Licona, G., \& Barquera, S. (2019). La obesidad en México. Estado de la política pública y recomendaciones para su prevención y control. Instituto Nacional de Salud Pública.

Ross, R., Neeland, I. J., Yamashita, S., Shai, I., Seidell, J., Magni, P., Santos, R. D., Arsenault, B., Cuevas, A., Hu, F. B., Griffin, B. A., Zambon, A., Barter, P., Fruchart, J. C., Eckel, R. H., Matsuzawa, Y., \& Després, J. P. (2020). Waist circumference as a vital sign in clinical practice: a Consensus Statement from the IAS and ICCR Working Group on Visceral Obesity. Nature Reviews Endocrinology, 16(3), 177-189. https://doi.org/10.1038/s41574-019-0310-7

San-Cristobal, R., Navas-Carretero, S., MartínezGonzález, M. Á., Ordovas, J. M., \& Martínez, J. A. (2020). Contribution of macronutrients to obesity: implications for precision nutrition. Nature Reviews Endocrinology, 16(6), 305-320. https://doi.org/10.1038/s41574-020-0346-8

Sánchez, E., Sánchez, M., Betriu, À., Rius, F., Torres, G., Purroy, F., Pamplona, R., Ortega, M., López-Cano, C., Hernández, M., Bueno, M., Fernández, E., Salvador, J., \& Lecube, A. (2020). Are Obesity Indices Useful for Detecting Subclinical Atheromatosis in a Middle-Aged
Population? Obesity Facts, 13(1), 1. https://doi.org/10.1159/000502696

Shamah-Levy, T., Campos-Nonato, I., Cuevas-Nasu, L., Hernández-Barrera, L., Morales-Ruán, M. del C., Rivera-Dommarco, J., \& Barquera, S. (2019). Sobrepeso y obesidad en población mexicana en condición de vulnerabilidad. Resultados de la Ensanut 100k. Salud Pública de México, 61(6), 852-865. https://doi.org/10.21149/10585

Shidfar, F., Alborzi, F., Salehi, M., \& Nojomi, M. (2012). Association of waist circumference, body mass index and conicity index with cardiovascular risk factors in postmenopausal women. Cardiovascular Journal of Africa, 23(8), 442-445. https://doi.org/10.5830/CVJA-2012038

Smith, S. C., \& Haslam, D. (2007). Abdominal obesity, waist circumference and cardiometabolic risk: Awareness among primary care physicians, the general population and patients at risk - The Shape of the Nations survey. Current Medical Research and Opinion, 23(1), 29-47. https://doi.org/10.1185/030079906X159489

Sniderman, A. D., Couture, P., Martin, S. S., DeGraaf, J., Lawler, P. R., Cromwell, W. C., Wilkins, J. T., \& Thanassoulis, G. (2018). Hypertriglyceridemia and cardiovascular risk: a cautionary note about metabolic confounding. Journal of Lipid Research, 59(7), 1266-1275. https://doi.org/10.1194/jlr.R082271

Stevens, J., Katz, E. G., \& Huxley, R. R. (2010). Associations between gender, age and waist circumference. European Journal of Clinical Nutrition, 64(1), 6-15. https://doi.org/10.1038/ejcn.2009.101

Valdez, R. (1991). A simple model-based index of abdominal adiposity. Journal of Clinical Epidemiology, 44(9), 955-956. https://doi.org/10.1016/0895-4356(91)90059-I

Wall-Medrano, A., Ramos-Jiménez, A., HernandezTorres, R. P., Villalobos-Molina, R., TapiaPancardo, D. C., Jiménez-Flores, J. R., MéndezCruz, A. R., Murguía-Romero, M., GallardoOrtíz, I. A., \& Urquídez-Romero, R. (2016). Cardiometabolic risk in young adults from 
northern Mexico: Revisiting body mass index and waist-circumference as predictors. $B M C$ Public Health, 16(236), 1-9. https://doi.org/10.1186/s12889-016-2896-1

Welch, G. W., \& Sowers, M. R. (2000). The interrelationship between body topology and body composition varies with age among women. Journal of Nutrition, 130(9), 2371-2377. https://doi.org/10.1093/jn/130.9.2371

Wilson, K. (2020). Obesity: Understanding Obesity. FP Essentials, 1(492), 11-18.

World Health Organization. (2008). Waist Circumference and Waist-Hip Ratio. Report of a WHO Expert Consultation. https://doi.org/10.1038/ejcn.2009.139

Ying, X., Song, Z. Y., Zhao, C. J., \& Jiang, Y. (2010). Body mass index, waist circumference, and cardiometabolic risk factors in young and middle-aged Chinese women. Journal of Zhejiang University: Science B, 11(9), 639-646. https://doi.org/10.1631/jzus.B1000105

Yoo, E. G. (2016). Waist-to-height ratio as a screening tool for obesity and cardiometabolic risk. Korean Journal of Pediatrics, 59(11), 425431. https://doi.org/10.3345/kjp.2016.59.11.425

Zhang, X., Shu, X. O., Gao, Y. T., Yang, G., Matthews, C. E., Li, Q., Li, H., Jin, F., \& Zheng, W. (2004). Anthropometric predictors of coronary heart disease in Chinese women. International Journal of Obesity, 28(6), 734-740. https://doi.org/10.1038/sj.ijo.0802634 